\section{ARTIFICIAL INTELLIGENCE-POWERED SPATIAL ANALYSIS OF TUMOR-INFILTRATING LYMPHOCYTES REVEALS IMMUNE-EXCLUDED PHENOTYPE RELATED TO APOBEC SIGNATURE AND CLONAL EVOLUTION OF CANCER}

${ }^{1}$ Chan-Young Ock*, 'Sanghoon Song, 'Gahee Park, ${ }^{2}$ Changhee Park, 'Soo Ick Cho, ${ }^{1}$ Seunghwan Shin, 'Yoojoo Lim, 'Wonkyung Jung, ${ }^{1}$ Heon Song, 'Jeongun Ryu, 'Minuk Ma, ${ }^{1}$ Seonwook Park, 'Sergio Pereira, 'Donggeun Yoo, 'Kyunghyun Paeng. 'Lunit Inc., Seoul, Korea, Republic of; ${ }^{2}$ Seoul National University Hospital, Seoul, Kosovo, Republic of

Background Little is known about bridging clonal heterogeneity into the resistance of immune checkpoint inhibitors (ICI). Recent reports showed that excluded tumor-infiltrating lymphocytes (TIL) into stroma assessed by an artificial intelligence (AI)-powered spatial TIL analyzer, Lunit SCOPE IO, was related to loss-of-heterozygosity of HLA genes which would be one of crucial resistance pathways of ICI. $^{1}$ In the current study, we hypothesized that Immune-excluded phenotype called by Lunit SCOPE IO would be related to clonal heterogeneity resulted from genome-wide accidents during early carcinogenesis which may cause an improper targeting of TIL for diverse clones with multiple genomic aberrations.

Methods For spatial TIL analysis, we applied Lunit SCOPE $\mathrm{IO}^{1}$ which automatically detects TIL and segmentizes cancer area and stroma, then it classified Immune phenotype of 1 mm2-sized grid in H\&E image. Inflamed score or Immuneexcluded score were defined as the proportion of Inflamed phenotype, which is high intra-tumoral TIL density, or Immune-excluded phenotype, which is exclusively high TIL density only in stroma, within a whole-slide image, respectively. We evaluated the correlation of Immune phenotype with APOBEC mutational signature by single-base substitution (SBS) signature 2 and/or SBS13, ${ }^{2}$ whole-genome doubling, and subclonal genome fraction which reflects intra-tumoral heterogeneity, ${ }^{3}$ and clusters of $\mathrm{T}$ cell receptor (TCR) repertoire ${ }^{4}$ derived from previous reports of The Cancer Genome Atlas (TCGA), consists of 7,467 tumor samples from 22 cancer types.

Abstract 830 Table 1 Correlation between immune phenotype and clonal evolution of cancer [* Median ( $95 \%$ confidence interval)]

\begin{tabular}{|c|c|c|c|c|}
\hline & & $\begin{array}{l}\text { Immune-excluded score } \\
\text { (Lunit SCOPE IO) }\end{array}$ & $\begin{array}{l}\text { Inflamed score } \\
\text { (lunit SCOPE IO) }\end{array}$ & $\begin{array}{l}\text { Immune cytolytic activity } \\
\text { (GZMA and } P R F 1 \text { ( }\end{array}$ \\
\hline $\begin{array}{l}\text { APOBEC mutational signature } \\
\text { (SBS2 and/or SBS13) }\end{array}$ & $\begin{array}{l}\mathrm{No*}^{*} \\
\text { Yes } \\
\text { Fold change } \\
\rho\end{array}$ & $\begin{array}{l}\text { (Lunit SCOPE IO) } \\
29.4 \%(2.94 .744 .4 \%) \\
37.8 \%(5.82-77.5 \%) \\
+28.6 \% \\
<2.6 \% 10.10\end{array}$ & $\begin{array}{l}\text { (Lunit SCOPE IO) } \\
17.3 \%(0.61-81.4 \%) \\
15.8 \%(0.54-75.9 \%) \\
-8.67 \% \\
0.00315\end{array}$ & 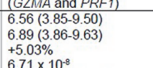 \\
\hline Whole genome-doubling & $\begin{array}{l}\text { No* } \\
\text { Yes* } \\
\text { Fold change } \\
\rho \text { P che }\end{array}$ & $\begin{array}{l}29.1 \%(2.99-72.9 \%) \\
38.3 \%(4.84-79.4 \%) \\
+31.6 \% \\
<2.70\end{array}$ & $\begin{array}{l}18.1 \%(0.64 .80 .5 \%) \\
14.2 \%(0.74 .2 \%) \\
-21.5 \% \\
-22 \times 10^{-16}\end{array}$ & $\begin{array}{l}6.71(3.87-9.62) \\
6.73(3.55-9.44) \\
+2.98 \% \\
0.466\end{array}$ \\
\hline $\begin{array}{l}\text { Subclonal genome fraction } \\
\text { (intra-tumoral heterogeneity) }\end{array}$ & $\begin{array}{l}<\text { Ledian* } \\
\text { zMedian" } \\
\text { Fold change } \\
\rho\end{array}$ & $\begin{array}{l}27.2 \%(2.61-71.2 \%) \\
38.0 \%(4.95-78.5 \%) \\
+39.7 \% \\
<22 \times 10.10\end{array}$ & $\begin{array}{l}16.5 \%(0.45-80.2 \%) \\
16.4 \%(0.53-77.2 \%) \\
-0.61 \% \\
0.419\end{array}$ & $\begin{array}{l}6.44(3.66-9.40) \\
6.91(4.05-9.69) \\
+7.30 \% \\
<22 \times 10^{-16}\end{array}$ \\
\hline
\end{tabular}

Results In the TCGA pan-carcinoma database, APOBEC mutational signature was significantly correlated with increased ratio of cancer stroma to cancer epithelium (median 0.866 vs 1.19 , fold change $+37.4 \%$ ), and increased TIL density in cancer stroma (median 558 vs $764 / \mathrm{mm} 2$, fold change $+36.9 \%$ ), but it was not correlated with intra-tumoral TIL density (median 63 vs $59 / \mathrm{mm} 2$, fold change $-6.3 \%$ ). Interestingly, Immune-excluded score (IES) called by Lunit SCOPE IO was positively correlated with APOBEC mutational signature as well as expression levels of APOBEC1, APOBEC3A, and APO$\mathrm{BEC} 3 \mathrm{~B}$, whole-genome doubling, and subclonal genome fraction, respectively, while Inflamed score (IS) or immune cytolytic activity (GZMA and PRF1 expressions) was negatively or not significantly correlated to those variables (table 1). TCR repertoire was expanded in the tumor samples with high IS (spearman rho $=0.279$ ), but it was not increased in those with high IES (spearman rho $=-0.0595$ ).

Conclusions There is a significant correlation between distinct TIL deposition in stroma, or Immune-excluded phenotype, with APOBEC-attributed clonal expansion of cancer, without proper expansion of TCR repertoire.

\section{REFERENCES}

1. Ock CY, Park C, Paeng K, Yoo D, Kim S, Park S, Lee SH, Mok T, Bang YJ. Artificial intelligence-powered spatial analysis of tumor-infiltrating lymphocytes reveals distinct genomic profile of immune excluded phenotype in pan-carcinoma. Cancer Res 2021;81(Supp 13):1908.

2. Alexandrov LB, Kim J, Haradhvala NJ, Huang MN, Tian Ng AW, Wu Y, Boot A, Covington KR, Gordenin DA, Bergstrom EN, Islam SMA, Lopez-Bigas N, Klimczak LJ, McPherson JR, Morganella S, Sabarinathan $R$, Wheeler DA, Mustonen $V$, PCAWG Mutational Signatures Working Group, Getz G, Rozen SG, Stratton MR, PCAWG Consortium. The repertoire of mutational signatures in human cancer. Nature 2020;578(7793):94-101.

3. Taylor AM, Shih J, Ha G, Gao GF, Zhang X, Berger AC, Schumacher SE, Wang $C$, Hu H, Liu J, Lazar AJ, Cancer Genome Atlas Research Network, Cherniack AD, Beroukhim R, Meyerson M. Genomic and functional approaches to understanding cancer aneuploidy. Cancer Cell 2018;33(4):676-689.e3.

4. Zhang H, Liu L, Zhang J, Chen J, Ye J, Shukla S, Qiao J, Zhan X, Chen H, Wu CJ, Fu YX, Li B. Investigation of antigen-specific T-Cell receptor clusters in human cancers. Clin Cancer Res 2020;26(6):1359-1371.

http://dx.doi.org/10.1136/jitc-2021-SITC2021.830 